\title{
SAW Investigation of Structural Changes in Oil-Based Magnetic Fluids
}

\author{
P. BurY ${ }^{a, *}$, F. Černobila ${ }^{a}$, M. VEVERIČÍK ${ }^{a}$, J. KÚdelČíK $^{a}$, \\ P. KOPČANSKÝ ${ }^{b}$, M. TIMKO ${ }^{b}$ AND M. RAJŇÁK ${ }^{b}$ \\ ${ }^{a}$ Department of Physics, Žilina University, Univerzitná 1, 01026 Žilina, Slovakia \\ ${ }^{b}$ Institute of Experimental Physics, Slovak Academy of Sciences, Watsonova 47, 04001 Košice, Slovakia
}

\begin{abstract}
Structural changes in transformer oil-based magnetic fluids under magnetic fields are investigated using surface acoustic waves. The measurement of the attenuation of SAW propagating along the interface between magnetic fluid and substrate is showed as an effective tool to study such processes in the cases when the obviously utilized longitudinal acoustic waves due to the higher viscosity are not available. The magnetic particles $\mathrm{FeO} \cdot \mathrm{Fe}_{2} \mathrm{O}_{3}$ in several different volume concentrations were added to the transformer oil MOL. The interactions between magnetic field and magnetic moments of nanoparticles lead to the aggregation of magnetic nanoparticles and subsequent chain or cluster formations that have the influence on the SAW attenuation. Temperature of magnetic fluids has also very important influence on structural changes due to the change of viscosity and thermal motion with increasing temperature that both affect the process of cluster creation. The measurement of the acoustic anisotropy gives additional useful information about the structure of nanoparticles formations.
\end{abstract}

DOI: 10.12693/APhysPolA.137.964

PACS/topics: magnetic fluids, structural changes, SAW

\section{Introduction}

Magnetic fluids (MFs) have found wide application in technology $[1,2]$ due to new interesting physical properties. The addition of magnetic nanoparticles to the transformer oils can improve their properties which is interesting also from the point of view of power electronics. The transformer oil-based MFs can then have better thermal and even insulating properties than transformer oils in pure form usually used for both high voltage insulation and power electronics cooling. However, their dielectric breakdown strength can be strongly influenced by the aggregation of magnetic nanoparticles in magnetic field and can induce electric breakdown $[3,4]$. The structural properties of the MFs under external magnetic field are affected by the orientation of magnetic moments of nanoparticles and their ability to shape up into some complex formations. An applied magnetic field induces ordering of the magnetic moments of the particles, giving rise to magnetization of the whole MF and thereby creating quasi spherical formations or even clusters [3-6]. The shape of such formations depends on the particle parameters, their concentration, the viscosity, and temperature of MF.

One of the useful methods to study the structure of MFs is based on the measurement of the attenuation of longitudinal acoustic wave $\alpha$ under an external magnetic field in different conditions $[7,8]$. The interaction between the acoustic waves and the magnetic nanoparticles or clusters leads to the additional attenuation of acoustic

\footnotetext{
*corresponding author; e-mail: bury@fel.uniza.sk
}

wave compared to that in the carried liquid. However, when the viscosity of magnetic fluid is too high, the measurement of acoustic attenuation is more difficult, and this technique cannot be utilized properly. Recently, we have shown that the surface acoustic waves (SAW) technique can be very useful for the investigation of structural changes in doped liquid crystals $[9,10]$. In this contribution, we utilized previous studies to investigate structural changes in MFs based on the transformer oil MOL doped with magnetic nanoparticles $\mathrm{FeO} \cdot \mathrm{Fe}_{2} \mathrm{O}_{3}$ that were added to the transformer oil in several different volume concentrations. Both linear increasing magnetic field and pulsed magnetic field were applied to study structural changes. The interaction between magnetic field and magnetic moments of nanoparticles led to the aggregation of magnetic nanoparticles that have the influence on the SAW attenuation.

\section{Experimental}

The investigated MFs are based on inhibited insulating oil TO $40 \mathrm{~A}$ MOL. The important parameters of MOL are: density of $867 \mathrm{~kg} / \mathrm{m}^{3}$ (at $15^{\circ} \mathrm{C}$ ), pour point at $-45^{\circ} \mathrm{C}$, flash point at $140^{\circ} \mathrm{C}$, and dynamic viscosity of $22 \mathrm{mPa} s\left(\right.$ at $\left.20^{\circ} \mathrm{C}\right)$. The dispersed phase was composed of co-precipitated iron oxide $\left(\mathrm{FeO} \cdot \mathrm{Fe}_{2} \mathrm{O}_{3}\right)$ nanoparticles stabilized with oleic acid (surfactant). The density of MFs was $991 \mathrm{~kg} / \mathrm{m}^{3}$ for $3.5 \%$ of volume concentration. The magnetization of saturation was $175 \mathrm{~A} \mathrm{~m}^{2} / \mathrm{kg}$ and the mean diameter of the particle was $122 \mathrm{~nm}$ with a deviation of $63 \mathrm{~nm}$.

The SAW of frequency of $10 \mathrm{MHz}$ was generated by the interdigital transducer that was prepared on the $\mathrm{LiNbO}_{3}$ delay line, using the Pulse Modulator and ReceiverMATEC 7700. Another transducer was used for receiving 


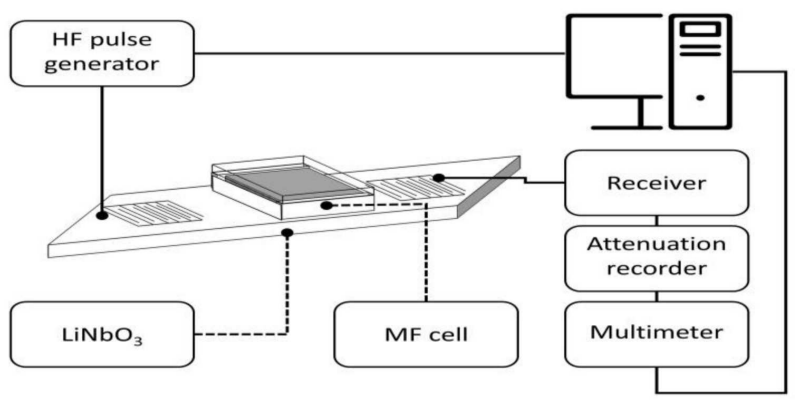

Fig. 1. Schematic illustration of experimental arrangement.

the surface wave (Fig. 1). The SAW attenuation was measured using MATEC Attenuation Recorder 2470 A. The cell with magnetic fluids was placed at the center of the top of the $\mathrm{LiNbO}_{3}$. When the SAW propagates along the delay line that is in contact with MF, SAW radiates a longitudinal wave into the $\mathrm{MF}$, giving rise to the propagation loss. This mode conversion is caused by the vertical surface displacement of SAW [11]. The shear displacement at the surface drives shear fluid movement that results in the additional increase in the propagation loss. The interaction of both modes of SAW with MF layers can cause the rise or decrease of the SAW attenuation when MF nanoparticles change their orientation and create structures due to the change in external conditions.

\section{Results and discussion}

The SAW generated on a solid substrate propagates along the surface as an elliptically polarized wave, with the displacement amplitude decaying into the substrate. Upon reaching an MF, the SAW amplitude is exponentially damped. It propagates as a leaky surface wave at the fluid/substrate interface due to the generation of a longitudinal wave into the fluid that itself propagates at the Rayleigh angle $\emptyset_{R}$ [11]. The interaction of these SAW modes with MF layers then can increase or decrease the SAW attenuation when magnetic particles and/or carrier liquid change their properties due to the change in external conditions.

The dependence of the SAW attenuation on linearly increasing external magnetic field and constant temperature of MFs is shown in Fig. 2. This figure presents the attenuation changes for magnetic field $\boldsymbol{B}$ perpendicular to $\boldsymbol{k}$, for transformer oil MOL with two different concentrations of magnetic nanoparticles, MOL $3(1.5 \%)$ and MOL $4(2 \%)$. The SAW attenuation changes in MOL 2 (1\%) are not illustrated in the figure because they were negligible at temperature of $25^{\circ} \mathrm{C}$. It shows the important role of nanoparticle concentration in this type of transformer oil based MFs. When the magnetic field after reaching the maximum value decreased to zero field, a steady increase up to $\approx 100 \mathrm{mT}$ and residual attenuation at zero field.

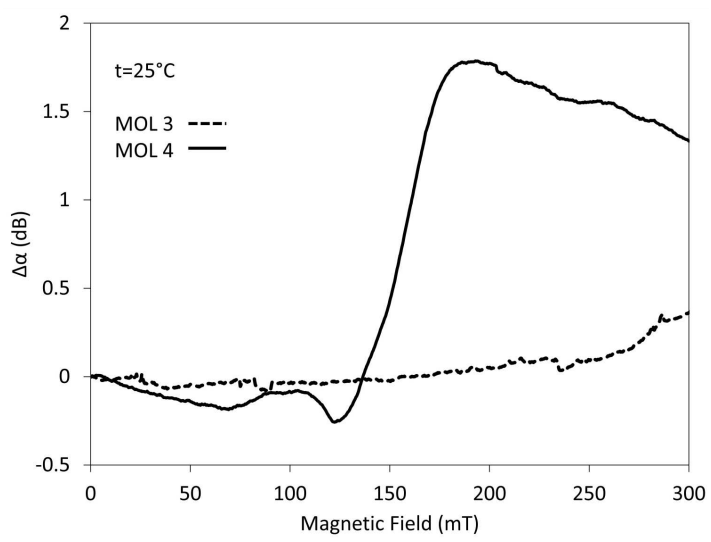

Fig. 2. Dependence of SAW attenuation changes on external magnetic field for MFs Mol 3 and Mol 4 measured at $25^{\circ} \mathrm{C}$.

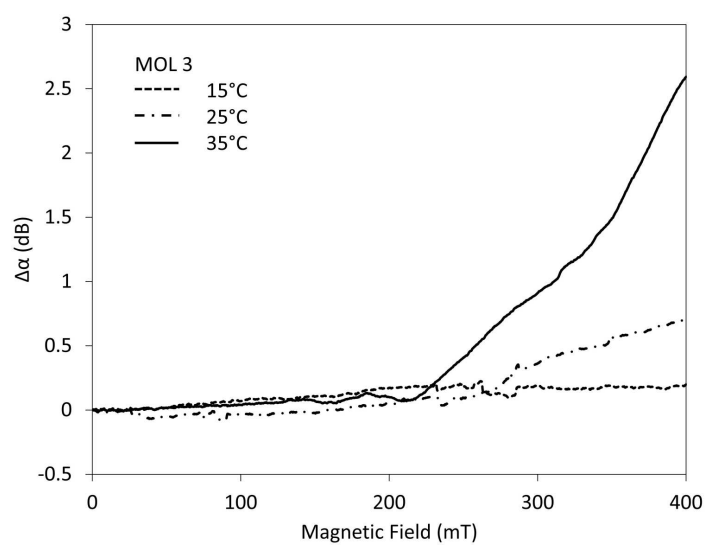

Fig. 3. As in Fig. 2, but measured at different temperatures.

Figure 3 illustrates the dependence of the SAW attenuation on increasing magnetic field and constant rate measured for MOL 3, as a representative MF, measured at three different temperatures: 15,25 , and $35^{\circ} \mathrm{C}$. As it can be seen, the high viscosity at low temperatures does not allow the formation of some spherical formations or even clusters. The role of lowering of viscosity with increasing temperature predominate over the role of thermal motion.

Figure 4 illustrates the SAW attenuation behavior for pulsed changes of the magnetic field for MOL $4(2 \%)$ as representative sample. On the obtained development three different ranges of the SAW attenuation progress can be distinguished, relatively fast increase, followed by slow increase and finally saturation. Besides the SAW attenuation decreasing at $15^{\circ} \mathrm{C}$ to the original value after the magnetic field was removed, its measurement at temperatures higher than $25^{\circ} \mathrm{C}$ shows the remanent attenuation that suggests creation of more complex forms of particles and their long lasting time duration. So when the magnetic field was switched off, the SAW attenuation did not return to the initial value, which means that most clusters still remained together in the MF. 


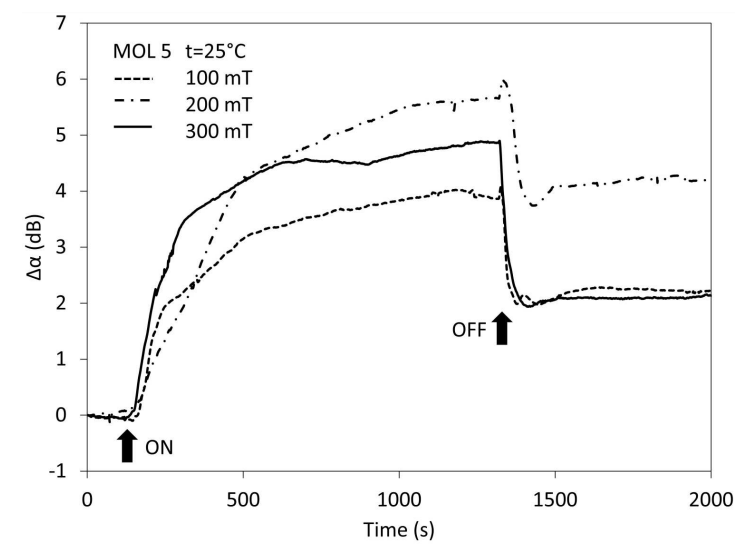

Fig. 4. Time development of SAW attenuation for different pulsed changes of magnetic field $(200,300$, and $400 \mathrm{mT}$ ) observed for MOL 4 and measured at temperature $15^{\circ} \mathrm{C}$.

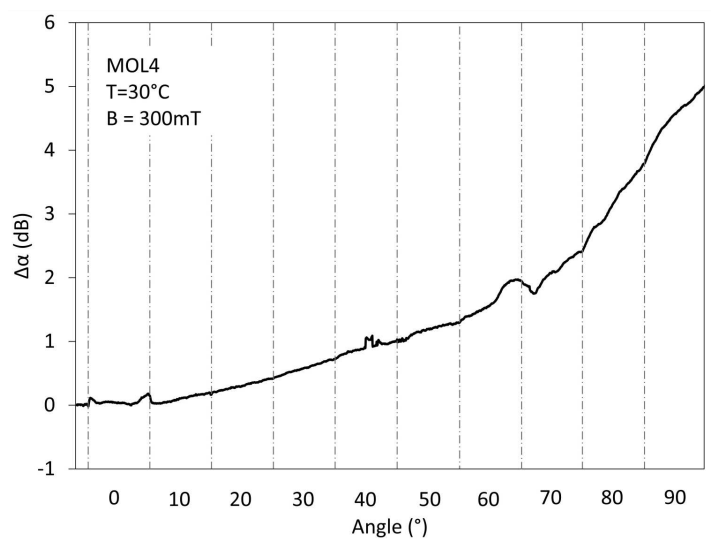

Fig. 5. (a) Anisotropy of the SAW attenuation $(B=300 \mathrm{mT})$ measured for magnetic fluid MOL 4 at $30^{\circ} \mathrm{C}$. External magnetic field $\boldsymbol{B}$ was parallel at $0^{\circ}$ and perpendicular at $90^{\circ}$ to wave vector $\boldsymbol{k}$.

Applying the theoretical predictions of Taketomi [5] to our results of anisotropy measurement (Fig. 5), the translational motion of nanoparticles dominates under the effect of acoustic wave, and the rotational motion can be neglected. This is close to the theory of the Shliomis et al. [6], that predicted only translational and vibrational motions. If we then take into account the fact that the rotational motion of clusters is very important for larger forms and/or clusters eventually at higher coupling constant, then the obtained results indicate shorter size of created nanoparticle formations or their lower volume concentration in investigated MFs.

\section{Conclusions}

The acoustic spectroscopy utilizing SAWs as a new technique for the study of structural changes of MFs is presented. The effect of magnetic field on the creation of new structures from magnetic nanoparticles was investigated in MF based on the transformer oil MOL characterized by higher viscosity. The results pointed out the extremely important role of concentration and temperature on structural changes in investigated MFs, represented here by SAW attenuation changes. The strong influence of magnetic field on the MFs structure was observed only at higher temperature, over $30^{\circ} \mathrm{C}$, due to the decreasing viscosity, and for highest concentration. The observed behavior of the SAW attenuation validated the process of aggregation of magnetic particles into complex form, however, their sizes are shorter. The anisotropy measurement showed also the important role of the translational motion of created forms to the SAW attenuation. Obtained results unambiguously approved the SAW technique as a useful method for MFs investigation.

\section{Acknowledgments}

This work was supported by VEGA projects $2 / 0016 / 17,1 / 0510 / 17$.

\section{References}

[1] V. Segal, K. Raj, Indian J. Eng. Mater. Sci. 5, 41 (1998).

[2] S. Odenbach, Coll. Surf. A Physicochem. Eng. Asp. 217, 171 (2003).

[3] P. Kopčanský, K. Marton, M. Koneracká, M. Timko, I. Potočová, J. Magn. Magn. Mater. 272, 2377 (2004).

[4] J. Kúdelčík, P. Bury, P. Kopčanský, M. Timko, Phys. Proced. 9, 78 (2010).

[5] S. Taketomi, J. Phys. Soc. Jpn. 55, 838 (1986).

[6] M. Shliomis, M. Mond, Phys. Rev. Lett. 101, 074505 (2008).

[7] A. Józefczak, J. Magn. Magn. Mater. 293, 240 (2005).

[8] J. Kúdelčík, P. Bury, P. Kopčanský, M. Timko, J. Magn. Magn. Mater. 326, 75 (2013).

[9] P. Bury, J. Kúdelčík, Š. Hardoň, M. Veveričík, P. Kopčanský, M. Timko, V. Závišová, J. Magn. Magn. Mater. 423, 57 (2017).

[10] P. Bury, M. Veveričík, J. Kúdelčík, P. Kopčanský, M. Timko, V. Závišová, Acta Phys. Pol. A 131, 913 (2017).

[11] B. Tiller, J. Rebous, M. Tassieri, R. Wilson, J.M. Cooper, Phys. Fluids 29, 122008 (2017). 